\section{El desafío de construcción de una política de promoción de la Economía Social y Solidaria. El caso del gobierno de la provincia de Santa Fe}

\section{Mauro Casella}

Subsecretario de Economía Social

del Ministerio de Desarrollo Social

del gobierno de la provincia

de Santa Fe, Argentina
Economía Social y Solidaria /

Desafíos de gestión

RECEPCIÓN: 26/06/15

ACEPTACIÓN FINAL: 10/09/15

\section{Resumen}

Las políticas de promoción de la Economía Social y Solidaria (ESS) encarnan grandes desafíos para los Estados provinciales que transitan entre la falta de presupuesto, una institucionalidad escasa y capacidades deficientes para el desarrollo de tales políticas. El caso del Gobierno de Santa Fe representa un ejemplo de abordaje particular donde el eje estuvo puesto en ganar institucionalidad, escala y capacidades para afrontar los desafíos de promover una política provincial de fortalecimiento de la ESS.

\section{Palabras clave}

- Economía Social y Solidaria

- políticas públicas

- desarrollo territorial

\section{Resumo}

As políticas de promoção da Economia Social e Solidária (ESS) encarnam grandes desafios para os Estados provinciais que acontecem entre a falta de verbas, uma fraca institucionalidade e capacidades insuficientes para o desenvolvimento de tais políticas. O caso do Governo de Santa Fe representa um exemplo de abordagem específica onde o eixo foi definido em ganhar institucionalidade, escala e recursos para enfrentar os desafios de promover uma política provincial de fortalecimento da ESS

Palavras-chave

- Economia Social e Solidária

- políticas públicas

- desenvolvimento territorial

Para citación de este artículo

Casella, M. (2015). El desafío de construcción de una política de promoción de la Economía Social y Solidaria. El caso del

Gobierno de la Provincia de Santa Fe. En Revista +E versión digital, (5), pp. 142-149. Santa Fe, Argentina: Ediciones UNL. 
desde esa perspectiva, la ESS no
se plantea como una economía de
y para los pobres sino como una
propuesta para todos los ciudadanos
"Adoptar la lógica del Desarrollo Humano Sustentable puede tener corolarios sorprendentes: no se trata de competir incondicionalmente por el capital, acomodando la sociedad y el estado a su lógica sino de competir por la gente, de convertirse en un territorio atractivo para vivir como ciudadanos cabales" Coraggio, José Luis (2001).

\section{Palabras preliminares}

El gran desafío a partir de la segundo período del Frente Progresista Cívico y Social al frente del Gobierno de la provincia de Santa Fe (2011-2015) fue construir una institucionalidad y herramientas de gestión política que permitan dotar de escala e instalar en la agenda a la Economía Social y Solidaria (ESS) dando visibilidad a la gran diversidad de actores, acciones y procesos que la constituyen y representan a lo largo y ancho del territorio provincial.

Si bien la temática de la ESS fue abordada desde 2003 sin un área específica, el primer paso en la institucionalización de la misma hacia el interior del gobierno provincial fue durante la primera gestión del Frente Progresista, cuando las distintas experiencias de ESS existentes estaban bajo la órbita de dos direcciones, a saber, la Dirección de Inclusión Socioproductiva y la Dirección de Economía Social y Solidaria.

Este antecedente no logró escala provincial puesto que sus acciones estuvieron centradas más en las experiencias que en "lo territorial", no logrando articular una política provincial de promoción de la ESS pero sí dejando un antecedente desde el cual poder abordarla. En tanto, a nivel territorial, diversas organizaciones y cooperativas con peso propio reclamaban políticas para el sector tomando como antecedente lo realizado en la ciudad de Rosario a través de la Subsecretaría de Economía Solidaria local.

Por su parte, desde las universidades, específicamente desde la Universidad Nacional del Litoral, se estableció como materia optativa en la Facultad de Ciencias Económicas la cátedra de ESS y en la Universidad Nacional de Rosario la maestría en Entidades de la Economía Social.
Fue recién a partir de 2011, con la creación de la Subsecretaría de Economía Social en el ámbito del Ministerio de Desarrollo Social, que el sector contó con un área de escala y alcance provincial para el desarrollo de políticas específicas.

En el marco de la reconfiguración institucional que significó la creación de la Subsecretaría de Economía Social, se propuso extender territorialmente las políticas de ESS articulándose con aquellas experiencias socioproductivas existentes de modo disperso en el territorio provincial y, paralelamente, propiciando el surgimiento de otras nuevas en aquellos lugares donde las mismas eran inexistentes aprovechando la institucionalidad creada. Se asumió esta tarea desde una lógica de intervención y articulación que, alejándose de perspectivas asistencialistas, pretendió acompañar a los emprendimientos y promover la ESS mediante el fortalecimiento de diálogos, acuerdos y compromisos entre los distintos actores involucrados y la difusión de valores, tales como, reciprocidad, solidaridad, asociatividad, cooperación, preservación del medio ambiente y democratización de las prácticas socioeconómicas. Esta nueva lógica significó profundos y continuos cambios institucionales y de gestión.

Los objetivos de la nueva Subsecretaría están en diálogo con un campo de reflexión que en los últimos diez años se dio en el ámbito académico intentando analizar dos procesos simultáneos: el diseño de políticas públicas orientadas a la promoción del empleo autogestivo y el trabajo asociativo, por un lado, y el creciente impulso de experiencias diversas de autogestión y gestión colectiva del trabajo por parte de organizaciones, cooperativas y emprendedores, por otro.

En este marco, Coraggio plantea la potencialidad de estas experiencias para crear un sector de la ESS orientado hacia la reproducción ampliada de la vida del conjunto de la sociedad y no exclusivamente a la acumulación de capital, apuntando hacia la centralidad del trabajo y poniendo al hombre y su actividad por sobre las cosas y su valor monetario (Coraggio, en Guerra, 2007). Desde esa perspectiva, la ESS no se plantea como una economía de y para los pobres sino como una propuesta para todos los 
ciudadanos que, igualmente, intenta asegurar la inclusión de los pobres, de los excluidos. Esto significaría el reencastramiento de la economía en una sociedad en proceso de transformación progresiva o, en otros términos, la economía produciendo otra sociedad más justa e igualitaria (Hintze, 2007). O, en términos de Coraggio (2002), esta producción de sociedad es posible porque se vuelve a unir producción y reproducción, pues se produce para satisfacer de manera más directa y mejor las necesidades acordadas como legítimas por la misma sociedad.

En estas propuestas además de la centralidad del trabajo cobran vital importancia las dimensiones de:

- Territorio: es crucial en esta propuesta el fortalecimiento de los territorios, entendiendo con ello la densidad social y organizativa de los mismos. En el territorio se articulan los actores y recursos involucrados en las políticas de la ESS.

- Autogestión: íntimamente asociada a la dimensión anterior, la autogestión supone la articulación de los trabajadores entre sí y de estos con los diferentes niveles de gobierno. La construcción de lazos asociativos y el fortalecimiento de acuerdos territorializados son claves en el proceso de empoderamiento de los emprendedores. - Sustentabilidad: entendida como sostenibilidad socioeconómica que, sin embargo, admite subsidios económicos y también aportes de trabajo u otros recursos no computados como costos (Coraggio, 2005).

\section{La Subsecretaría de Economía Social del Gobierno de Santa Fe. Construyendo políticas}

En diálogo con estos aportes teóricos, desde la Subsecretaría se considera que la ESS es una construcción cuyos horizontes y condiciones (sus puntos de llegada y de partida) deben ser discutidos, consensuados entre los diferentes actores involucrados y articulados con la realidad concreta de cada territorio. En otros términos, antes que ceñirse a "modelos" sobre lo que la economía "debería ser", este marco conceptual permitió trazar algunas líneas de acción que orientaron el trabajo de la Subsecretaría en constante diálogo con las experiencias concretas y la situación particular del territorio provincial, donde la ESS se materializó de un modo específico.

Por ello, los dos grandes objetivos en esta etapa de institucionalización de la ESS fueron:

- generar procesos de inclusión social y desarrollo territorial a través del estímulo de las capacidades locales y los recursos territoriales disponibles, promoviendo los vínculos solidarios y asociativos para pasar de la acción microsocial al desarrollo de una economía del trabajo;

- propiciar el desarrollo de capacidades e instrumentos para la gestión de la ESS en municipios y comunas y organizaciones sociales de apoyo y/o intermedias.

En función de los dos grandes objetivos se diseñaron estrategias que, si bien se presentan por separado, están estructuradas sobre la base de una misma política:

1- La extensión territorial y la agenda de la Economía Social en el territorio provincial.

2- La construcción del capital social, entendiendo con ello el fortalecimiento de los lazos sociales, el asociativismo y la solidaridad, por un lado y la autogestión por otro.

En cuanto a la extensión territorial de las políticas de la Subsecretaría (escala), vemos que en los cuatro años de gestión se llegó a 111 localidades (con las diferentes herramientas de apoyo, principalmente con los planes operativos marcados con color negro en el Mapa 1, lo cual fue acompañado con el presupuesto destinado al área. A continuación, en el cuadro 1, se muestra el desarrollo presupuestario de la Subsecretaría entre los años 2012 y 2015 a fin de presentar mediante datos concretos el proceso de promoción y desarrollo de la ESS como política estatal en la provincia de Santa Fe.

Como se observa en el Cuadro 1, entre los años 2013 y 2015 se registró un crecimiento sustantivo de los fondos dirigidos a la puesta en marcha de proyectos socioproductivos en el territorio provincial que, como veremos luego (Mapa 1), se reflejó en un aumento significativo de localidades alcanzadas por el Programa de Fortalecimiento a la ESS sumando un presupuesto ejecutado total, hasta el 30 de julio de 2015, de $\$ 30350804$. Es importante remarcar que desde 2012 a 2015 el presupuesto se incrementó un $400 \%$. Este incremento presupuestario se evidencia en el desarrollo territorial de la Subsecretaría (Mapa 1), lo que significa una escala que comprende a más de 6500 emprendedores, 87 cooperativas de trabajo, 65 organizaciones sociales, las ONG y asociaciones y 111 municipios y comunas que implementaron proyectos o planes operativos de fortalecimiento de la ESS, cumpliendo así las metas de brindar escala provincial y agendar la temática a nivel local. En cuanto a la construcción del capital social y capacidades en los territorios, podemos ver que en la extensión provincial existen más de 180 procesos asociativos contando aquellos que se dieron en función de la producción (cooperativas de trabajo y espacios colectivos de producción gestionados por municipios o comunas u
1) La campaña "Justo Lo Que Necesito" (JLQN) es parte de la estrategia de la Subsecretaría para promover el comercio justo y el consumo respon- sable. La misma se diseñó como una marca para identificar y sensibilizar en la materia a emprendedores y consumidores. 
organizaciones de la sociedad civil) y aquellos que se conformaron alrededor de las políticas de comercialización o circulación de bienes o servicios de la economía social apuntalados por la campaña "Justo Lo Que Necesito" principalmente traducidos en más de 80 espacios de ferias locales que tienen una continuidad en el tiempo y una organización estable muchas veces fomentada desde los Estados locales.

Esta gran red de trabajo territorial es sostenida a través del programa "Formador de formadores", ${ }^{2}$ destinado a promotores de la ESS, los cuales son financiados en cada uno de los Planes Operativos de Acción (POA) ${ }^{3}$ que implementan municipios y comunas.

A partir de la evaluación efectuada por la Subsecretaría, junto al Instituto Provincial de Estadísticas y Censos, acerca de la implementación de sus políticas, puede decirse que el $86 \%$ de los destinatarios de los proyectos (emprendedores, cooperativas, grupos asociativos) participó de instancias de capacitación y seguimiento promovidas a través del programa.

Otro de las estrategias para la construcción de capital social fue la promoción de instancias colectivas de producción y espacios de comercialización. En este sentido, el 62 \% de los destinatarios de los proyectos manifestó que el espacio por excelencia para comercializar es la Feria Local (Gráfico 1).

\section{Cuadro 1}

Montos financiados a proyectos y planes operativos en municipios, comunas y organizaciones por año por cada Nodo/Región

\begin{tabular}{|c|c|c|c|c|}
\hline Nodo/Año & 2012 & 2013 & 2014 & 2015 \\
\hline Rafaela & 161871 & 200943 & 566988 & 565723 \\
\hline Reconquista & 408644 & 806178 & 1178297 & 1025878 \\
\hline Rosario & 1268097 & 2829705 & 5249106 & 5152171 \\
\hline Santa Fe & 823318 & 1428606 & 3713149 & 3395795 \\
\hline Venado Tuerto & 108168 & 279044 & 533077 & 612564 \\
\hline Total & 2770097 & 5587957 & 11240619 & 10752131 \\
\hline
\end{tabular}

Fuente: elaboración propia, calculado sobre la base del presupuesto comprometido al 30/7/2015

Asimismo, una tendencia que mostró un crecimiento sostenido en los últimos años ha sido la utilización de Internet, y más particularmente las redes sociales, para promocionar y comercializar los productos y servicios de la ESS. En consecuencia, más del $50 \%$ de los emprendimientos relevados encontró allí una nueva herramienta que favorece la puesta en circulación de sus productos.

\section{Mapa 1}

Localidades donde se implementa el Programa de Fortalecimiento de la ESS

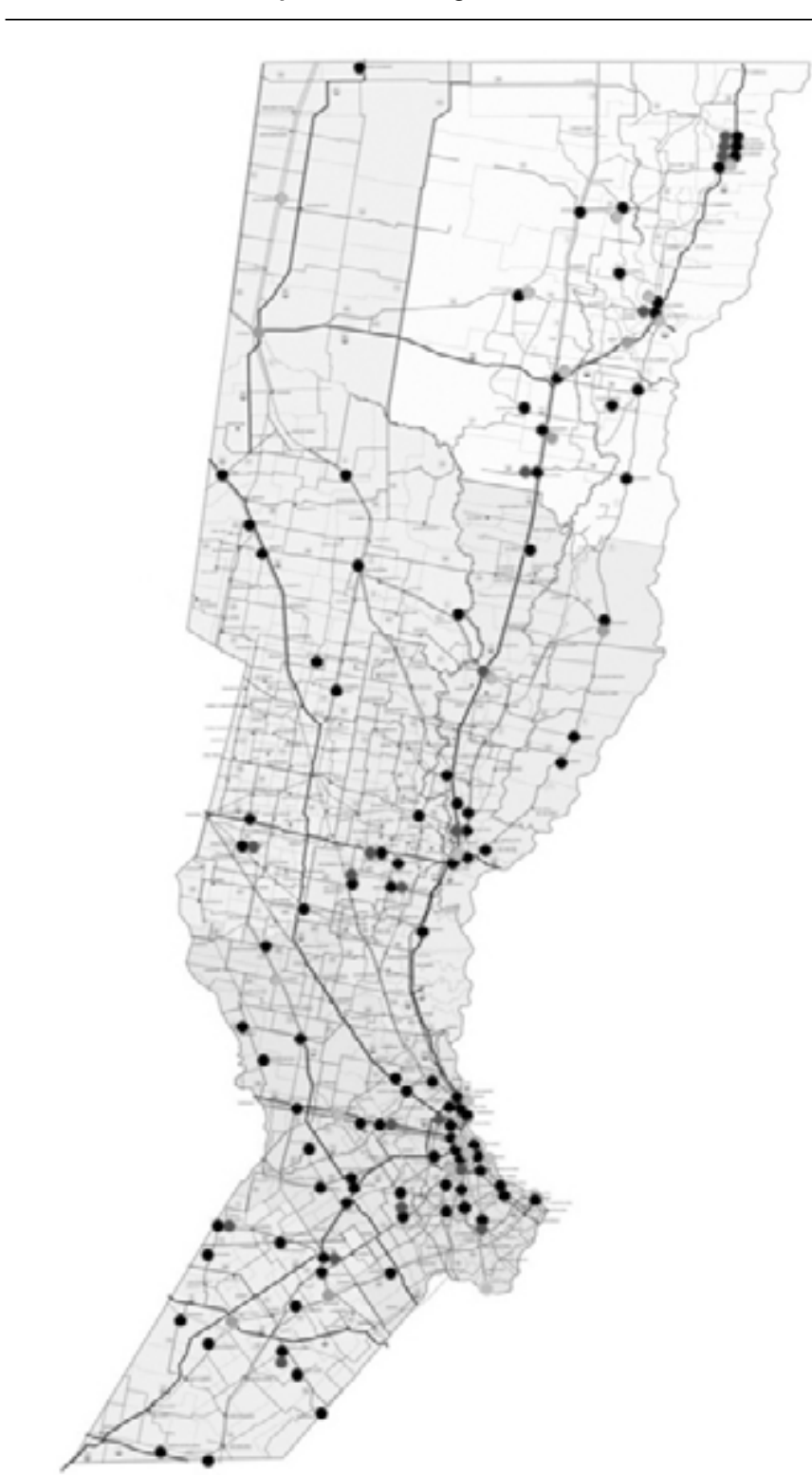

Ref::

- localidades con convenio

- localidades con proyectos de seguridad alimentaria

proyectos Pueblos Originarios

- fortalecimiento Organizaciones Sociales y Cooperativas

Fuente: Subsecretaría Economía Social.
2) El Programa Formación de Formadores está destinado a los Promotores de la ESS que se desempeñan en los municipios y comunas con proyectos financiados por la Subsecretaría. El objetivo de este programa es generar capacidades locales de acompañamiento y asistencia a los emprendedo- res a través de la formación continua de aquellos que están a cargo de las áreas locales o de la ejecución de los proyectos de fortalecimiento de la ESS.
3) El POA es una herramienta de planificación local que diseña cada municipio y comuna para ser financiado por la Subsecretaría de Economía Social. 


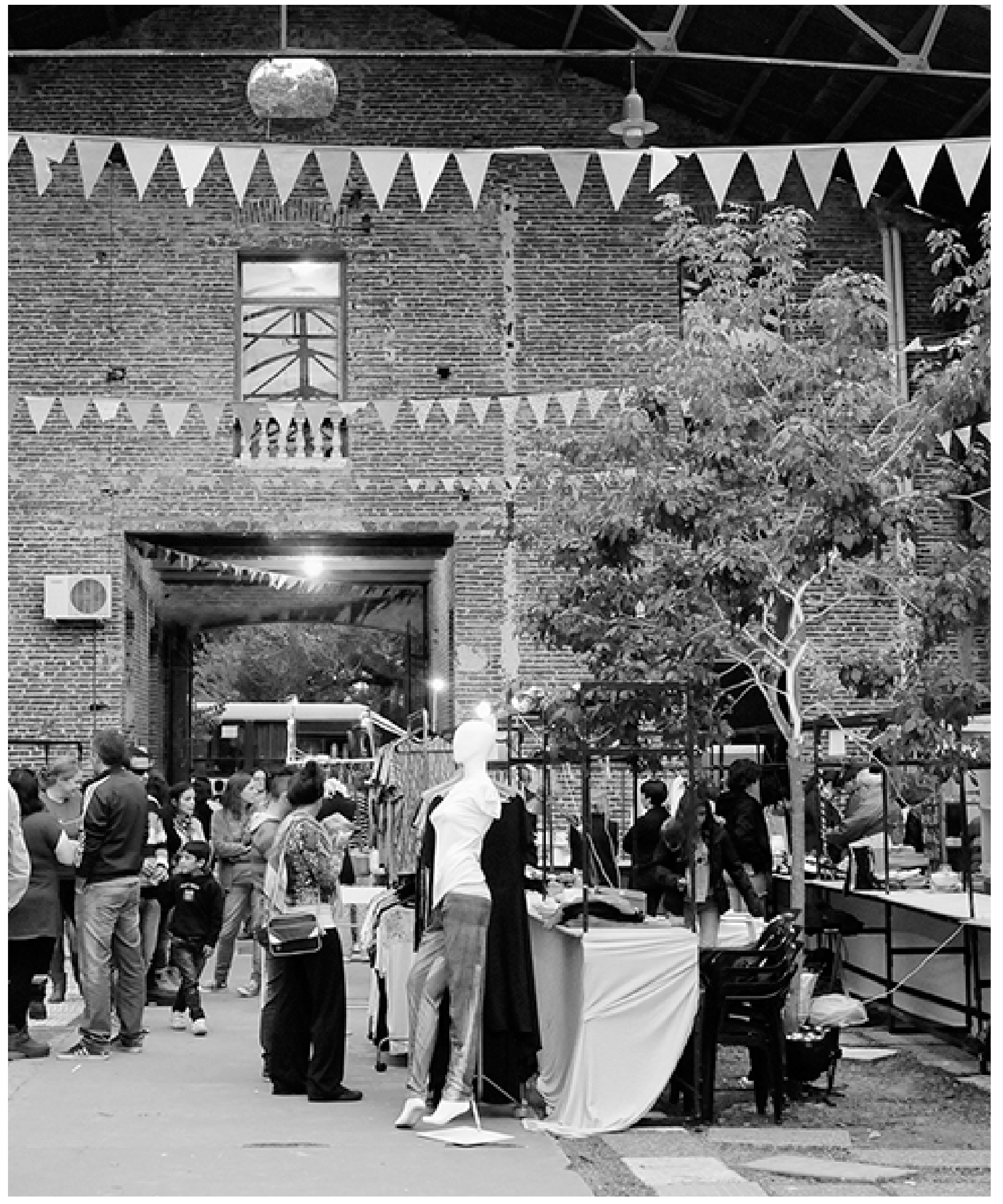


En cuanto a la promoción local de los espacios de comercialización, en el $43 \%$ de las localidades consultadas se realizan ferias mensualmente; mientras que en un $32 \%$ de las localidades se organizan con una frecuencia mayor (semanal o quincenal) y en el restante $25 \%$ con una frecuencia menor (bimestral, trimestral, etcétera). Otro dato significativo a la hora de evaluar estos espacios de encuentro y comercialización con relación al intercambio y la construcción de capital social puede verse en el fluido intercambio entre emprendedores de municipios y comunas cercanos: en el $90,9 \%$ de los casos relevados se reciben emprendedores de otras localidades en las ferias locales.

En sintonía con ello, respecto de qué porcentaje de emprendedores de las comunas y municipios relevados se movilizan para participar de las ferias organizadas en otras localidades, el mismo asciende a $85,3 \%$.

Es importante destacar que el programa "Justo Lo Que Necesito", adoptado en el $56 \%$ de las localidades, que tiene por objetivo promover los productos de la economía social y también los valores y criterios que sustentan su producción y consumo, posibilitó a través de campañas en estos espacios de comercialización que el 94,1\% de los actores de la ESS que participan lo conozcan y difundan. Por último, a la hora de evaluar la construcción de capital social, puede decirse que las políticas de promoción han logrado fortalecer

\section{Gráfico 1}

Espacios de comercialización

$\begin{array}{ll}\square \text { ferias locales } & \square \text { a pedido } \\ 62 \% & 8 \% \\ \begin{array}{ll}\text { ferias provinciales } \\ 18 \%\end{array} & \square \text { puerta a puerta } \\ & 2 \% \\ \text { comercios locales } & \square \text { otro } \\ 6 \% & 1 \% \\ & \end{array}$

Fuente: Subsecretaría Economía Social, 2015.

\section{Gráfico 2}

Espacios de producción colectiva en municipios y comunas promovidos por el Programa

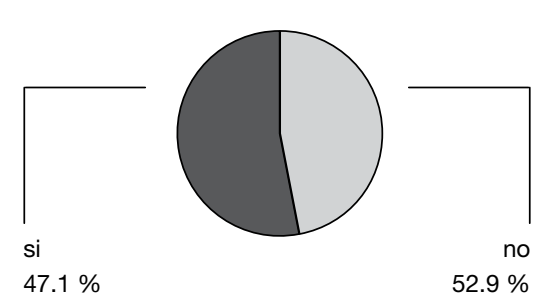

Fuente: Subsecretaría Economía Social, 2015. o generar espacios colectivos de producción en el $47 \%$ de las localidades (Gráfico 2), destacándose los espacios gestionados por los Estados locales (65\%) por sobre los espacios autogestivos, como cooperativas y asociaciones (17\%).

Si bien el fomento de la autogestión, el asociativismo y la cooperación forman parte de un proceso que implica modificar lógicas individuales profundamente arraigadas entre los sujetos a los que se dirigen (y sobre los cuales intervienen) las políticas implementadas por la Subsecretaría, es importante destacar que en más del $50 \%$ de las localidades aún no se ha avanzado en el fortalecimiento de los lazos asociativos, en tanto resulta satisfactorio (al mismo tiempo que alienta nuevos desafíos) que casi el $95 \%$ de los promotores reconozca que se han mejorado mucho $(50 \%)$ y bastante $(44,1 \%)$ los emprendimientos socioproductivos del territorio provincial a partir de las políticas de promoción.

\section{El camino hacia la institucionalización} a través de una Ley de Economía Social y Solidaria

En términos de institucionalización de las políticas de promoción de la ESS desde la Subsecretaría, se consideró prioritaria la sanción de una legislación provincial. Para ello se retomó un trabajo que incipientemente se estaba gestando desde la gestión anterior (2007-2011) junto a organizaciones sociales que venían planteando la necesidad de una legislación provincial.

Desde el año 2011 distintas organizaciones sociales, principalmente nucleadas en el Espacio Nacional de Economía Social y Solidaria (ENESS), plantearon la necesidad de contar con una ley provincial, para lo cual propusieron algunos lineamientos en un Foro provincial realizado en la ciudad de Rosario hacia fines de 2011.

Así pues, la iniciativa antecedió a la creación de la Subsecretaría que, sin embargo, asumió desde un principio el compromiso de darle continuidad y soporte a este proceso en marcha, con el objetivo de potenciar esas discusiones y organización previa y de fortalecer el diálogo entre el Estado y la sociedad civil. Con este espíritu, en el año 2012 se crearon las "Mesas de Trabajo Regionales", ${ }^{4}$ orientadas a acompañar y fortalecer las discusiones en torno a la ley.

De dichas Mesas participaron más de 55 organizaciones e instituciones que, hacia fines del año 2012, y como resultado del trabajo conjunto, lograron la presentación de los "Acuerdos básicos
4) Fueron instancias convocadas por la Subsecretaría de Economía social para darle escala provincial a las discusiones sobre la Ley de Economía Social y Solidaria. Dichos espacios fueron sostenidos y fomentados por la Subsecretaría, y a ellos asistieron organiza- ciones sociales, ONG y representantes de Estados Municipales y Comunales interesados en la temática. Las mismas funcionaron durante los años 2012 y 2013 hasta que las propias organizaciones constituyeron el Foro de la Economía Social y Solidaria de Santa Fe. 
para la construcción de una Ley de Economía Social y Solidaria en la Provincia de Santa Fe", en los cuales se sentaron los lineamientos que enmarcarían la discusión respecto de las características y objetivos del proyecto de ley.

Una vez acordados estos lineamientos, las organizaciones decidieron continuar de modo autogestivo la elaboración del proyecto de ley, constituyéndose el Foro de Economía Social y Solidaria que nuclearía tres Foros Regionales existentes en las ciudades de Santa Fe, Rosario y Reconquista. De algún modo, la construcción de un Foro Provincial es el resultado del intercambio y fortalecimiento de lazos propiciados en las Mesas de Diálogo.

Frente a ello, desde la Subsecretaría, lejos de desentenderse del proceso, continuó acompañándolo mediante el financiamiento de las actividades del Foro Provincial y promoviendo espacios de difusión en las Ferias Regionales de Economía Social. Y en el mes de noviembre de 2013 logró presentar el proyecto de la Ley de Promoción y Fomento de la Economía Social y Solidaria que, en líneas generales, recogió los fundamentos básicos presentes en los Acuerdos mencionados y aún continúa discutiéndose en la Legislatura provincial.

Este proceso no sólo es una experiencia fructífera de diálogo entre Estado y la sociedad civil (sin negar tensiones) sino que, a través del Foro de la Economía social y Solidaria, las organizaciones que antes no articulaban entre sí se constituyeron en un actor provincial con el cual discutir las políticas y acciones provinciales destinadas al sector.

\section{Articulaciones multinivel}

\section{para el fortalecimiento de la ESS a nivel local}

La articulación entre diferentes actores y niveles del Estado es esencial para lograr resultados en la promoción y el apoyo a la ESS. En este sentido, la experiencia del Centro de Emprendedores de la Economía Social (CEES) llevada adelante en la ciudad de Santa Fe es un ejemplo de articulación que puede destacarse como un aprendizaje a replicar en otras ciudades.

Se trata de un dispositivo local (funciona en la ciudad de Santa $\mathrm{Fe}$ ) donde se conjugan aportes de la UNL —a través de profesionales y tutores que acompañan y brindan capacitación a los emprendedores-, del Estado provincial -a través de la Subsecretaría de Economía Social que brinda aportes para proyectos de infraestructura, equipamiento y funcionamiento del Centro de Emprendedores así como también aportes para el financiamiento de proyectos de los emprendedores- y el Estado local - a través de la Dirección de Economía Social, que brindan capacidad operativa local, administración del espacio y ejecución de las actividades planificadas.

El Centro se coordina por medio de una mesa de gestión integrada por las tres partes intervinientes, las que planifican las acciones y marcan los objetivos y disponen de una unidad administrativa a cargo del gobierno local que ejecuta las actividades.

EI CEES es un centro de servicios destinados a emprendedores de la ESS donde se abordan temáticas que van desde la puesta en práctica de los valores que la constituyen, como la solidaridad y la reciprocidad, incentivando constantemente las prácticas asociativas y autogestivas, como la gestión de herramientas concretas de capacitación, acompañamiento, financiamiento y comercialización. EI CEES es. desde 2012, una referencia para los emprendedores de la ESS y cooperativas de la ciudad de Santa Fe, y ha logrado registrar casi 1000 emprendimientos y sostener un espacio permanente de formación y acompañamiento junto con la UNL, un espacio de comercialización semanal que se complementa con otros dispositivos de ferias itinerantes y un fondo rotatorio de financiamiento solidario aportado por el gobierno provincial de más de $\$ 350000$ que ha llegado a alrededor de 60 emprendimientos en los últimos tres años.

Además, se articularon junto al gobierno provincial dispositivos de inclusión socioproductiva en diferentes barrios de la ciudad de Santa $\mathrm{Fe}$, los que han logrado promover más de 150 emprendimientos. La contracara de este modelo de articulación es la relación entre los gobiernos provincial y nacional, donde ello no pudo lograrse ni en la implementación de fondos referidos a la Ley Nacional Microcrédito ni una coordinación efectiva de la implementación del Monotributo Social como forma de formalización de muchos emprendimientos.

En ambos casos, el gobierno nacional priorizó la implementación de dichas herramientas a través de los Centros de Referencia del Ministerio de Desarrollo Social de la Nación o de organizaciones sociales. Esto obligó a que los gobiernos locales debieran gestionar directamente estas herramientas. Asimismo, ello no impidió que el $79 \%$ de los municipios y comunas que implementaron el Programa de Fortalecimiento de la Economía Social realizara acciones tendientes a la formalización tributaria de sus emprendedores a través de la gestión del Monotributo Social. 


\section{Breves conclusiones}

Podemos concluir que, fundada en la estrategia planteada por la Subsecretaría para el cumplimiento de los objetivos propuestos al inicio de la gestión (2011-2015), se ha constituido una base territorial con capacidades de gestión y agendación de la temática que permiten visualizar algunos resultados en función de los procesos de inclusión social, visibilidad de los actores de la ESS y promoción de prácticas y valores que le son propios.

Sin dudas, los procesos más relevantes son las Mesas de Diálogo junto a organizaciones sociales que se han constituido como Foro Provincial y la cantidad de localidades que han logrado implementar planes y proyectos de fortalecimiento de la ESS, dos grandes hitos que permitirán, si la ley es sancionada, tener un sustento de desarrollo para dar cuerpo a las instituciones que se crean en la misma, para que sean protagonistas activos en los procesos de políticas públicas para el desarrollo social y económico local, promoviendo el trabajo asociativo, el cooperativismo, la agricultura familiar, el agregado de valor en origen, las cadenas productivas, el pleno respeto al uso del suelo y al cuidado del ambiente y finalmente, para que el Estado arbitre los mecanismos necesarios para brindar las herramientas: técnicas, económicas, de capacitación, de comercialización y de acceso a servicios. Este marco legal es un salto cualitativo en el proceso de institucionalización y formalización de la economía social en el territorio provincial que es necesario pero no imprescindible si en la próxima gestión (2015-2019) se profundizan algunos caminos transitados en el período analizado. Uno de los grandes desafíos de las próximas gestiones es no sólo lograr una institucionalización provincial (a través de la ley u otros mecanismos legislativos), sino dar institucionalidad a los procesos locales que fueron fortaleciéndose, tal como sucedió en la ciudad de Santa Fe a través del Centro de Emprendedores, en la ciudad de Rosario con la Subsecretaría de Economía Solidaria, y en Reconquista con la creación del área de ESS.

Lo destacable de este período es que, lejos de atarse a recetas o modelos preconstituidos, primaron la innovación, la promoción del diálogo, la búsqueda de consensos y de involucramiento por parte de los distintos actores (tanto estatales como de la sociedad civil), convencidos de que la ESS, antes que un punto de partida, es una construcción cotidiana, una invitación a pensar nuevas formas de desarrollo socioproductivo de los territorios, en torno al cual se vuelven ineludibles profundos cambios e innovaciones institucionales. Ello sabiendo que las prácticas asociativas, el cooperativismo, la autogestión, no se dan per se sino que suponen un trastocamiento de los modos en que se construyen las relaciones en el sistema capitalista; por lo que la invitación a pensar y diseñar colectivamente otros modos posibles de desarrollo socioproductivo, siempre en sintonía con la idiosincrasia y particularidades de los distintos territorios, permitió constituir una red para dar visibilidad a estas prácticas, lo que nos permite soñar que otro mundo es posible.

\section{Referencias bibliográficas}

Coraggio, J.L. (2001). Competir por el capital o competir por la gente? (230-251). En Vázquez Barquero, A. y Madoery, O. (comps.), Transformaciones globales, instituciones y políticas de desarrollo local. Rosario: Homo Sapiens.

Coraggio, J.L. (2002). La Economía Social como vía para otro desarrollo social. Documento de lanzamiento del debate sobre "Distintas propuestas de Economía Social". Urbared, Red de Políticas Sociales.

Coraggio, J.L. (2005). Sobre la sostenibilidad de los emprendimientos mercantiles de la economía social y solidaria. Seminario "De la Universidad Pública a la Sociedad Argentina. El plan Fénix en vísperas del segundo centenario. Una estrategia nacional de desarrollo con equidad". Universidad Nacional de Buenos Aires. Guerra, P. (coord.) (2007). ¿Cómo denominar a las experiencias económicas solidarias basadas en el trabajo? Diálogos entre académicos latinoamericanos sobre la polémica conceptual. En Otra Economía, I(1), 21-27.

Hintze, S. (2007). Políticas sociales argentinas en el cambio de siglo. Conjeturas de lo posible. Buenos Aires: Espacio Editorial. 